\title{
THE IMPACT OF PRURITUS ON QUALITY OF LIFE OF PATIENTS WITH LICHEN SIMPLEX CHRONICUS
}

\author{
Filka Georgieva1, Desislava Vankova² \\ ${ }^{1}$ Department of Dermatology and Venerology, Faculty of Medicine, \\ Medical University of Varna \\ ${ }^{2}$ ACRO, Medical University of Varna
}

\begin{abstract}
Lichen Simplex Chronicus (LSC) is a skin disorder affecting patient's psychosocial and functional status. Pruritus is a diagnostic hallmark for LSC. It is the reason for sleep disturbances, anxiety and depression. Therefore, a clinical investigation of pruritus alongside with a study of their self-reported health status is a necessary precondition for successful therapeutic results.

The aim of the study is to assess the impact of pruritus on quality of life (QoL) of LSC patients'.

MATERIALS AND METHODS: The presented study includes 190 out-patients diagnosed with LSC. Demographic data and disease-related characteristics were collected. Measurement tools, for assessing pruritus severity and sleep disturbance were visual analogue scale (VAS), and verbal rating scale (VRS).For the evaluation of QoL Dermatological Life Quality Index (DLQI) questionnaire was applied.

RESULTS AND DISCUSSION: Main complains of patients were pruritus and sleep disturbance. 40\% reported having low intensity pruritus (mean VAS 1.90), 36.84\% moderate pruritus (mean VAS 5.12), and 23.15\% severe pruritus (mean VAS 8.57). Results from sleep disturbance were: $4.73 \%$ reported currently having no sleep disturbance (mean VAS 0.18), $43.15 \%$ reported having mild sleep disturbance; $47.36 \%$ have moderate sleep disturbance (mean VAS 5.46), and 4.73\% severe sleep disturbance (mean VAS 8.93). The total DLQI score reported by the LSC-patients is 8.58 \pm 3.348 . Patients scores were significantly high for Q1 (symptoms), Q9 (sexual difficulties) and Q10 (treatment) $(\mathrm{p}<0=001)$.

CONCLUSION: All the reported and analyzed results indicate that pruritus is a major symptom in LSC and has a negative impact on all the QoL-domains for every patient $(\mathrm{p}<0.001)$. Consequently, pruritus moderately affects the QoL of the patients. This is the first study in our country, which aims to measure the impact of pruritus on QoL of LSH patients'.
\end{abstract}

Keywords: pruritus, quality of life, lichen simplex chronicus, Dermatology Life Quality Index

Address for correspondence:

Filka Georgieva

Department of Dermatology and Venerology

Medical University of Varna

9002 Varna

e-mail:filka@abv.bg

Received: May 4, 2016

Accepted: May 25, 2016

\section{INTRODUCTION}

Lichen Simplex Chronicus (LSC) is a common skin disorder characterized by lichenification of skin as a result of excessive scratching (1). LSC is distributed worldwide and affects adults with a mild prevalence for females (2). The pathogenesis of this dermatosis is not well distinguished. Disorders of skin barrier are described as a trigger or enhance pathological symptoms of LSC (3). On the other hand, psychological factors have an important role in the pathogenesis of LSC. Pruritus is the dominant symptom of LSC. 
Recent studies have indicated that this symptom has a major impact on health-related quality of life (4). The itch has been classified into four different clinical categories. These include neurogenic, psychogenic, neuropathic, and pruritoceptive (5). These categories were developed based on anatomical, pathophysiological, and psychological factors. Itch can be triggered by emotional stress or other underling pruritogenic stimuli. Recent evidence has indicated that psychological stress is associated with exacerbation of different skin conditions, including LSC. It has been proven that psychological disorders are closely connected with severity of pruritus sensation. Although neurodermatitis is not life threatening, it can produce an important psychosocial burden.

For the purpose of the study, the QoL-definition of World Health Organization (WHO) is accepted as a theoretical frame: "Quality of life is defined as individuals' perceptions of their position in life in the context of the culture and value systems in which they live and in relation to their goals, expectations, standards and concerns. It is a broad-ranging concept affected in a complex way by the persons' physical health, psychological state, and level of independence, social relationships and their relationship to salient features of their environment (6).

\section{THE AIM OF THE STUDY}

The aim is to assess the impact of pruritus on QoL of LSC patients' by evaluating the severity of pruritus and sleep disturbance with VAS, and VRS and compare it with the results from the answers to a standardized dermatology-specific quality of life instrument - DLQI (Specially answer to question1- symptoms).

\section{MATERIALS AND METHODS Settings and Sample}

The study was conducted among 190 nonhospitalized adult patients 106 female (55.78\%) and 84 male $(44.21 \%)$; at a mean age $57.17 \pm 14.45$ years; range 22-98 years) who visited the dermatology unit at "Sveta Anna" Medical Center Varna, within the period of January2013 and January 2015. The following inclusion criteria were applied: one or more lichen plaques, highly pruritic, accumulation of normal skin lines, excoriations. Individuals with psychiatric disorders and/or those using antidepressants have been excluded. Diagnosis was based on clinical observation and patient's history data.

\section{Methods}

To assess the intensity of pruritus VAS and VRS were used. The VAS, the most commonly used tool, to measure different symptoms as panic, depression, fatigue or pain was developed in 1921 by Hayes \& Patterson (7). It is a $10-\mathrm{cm}$ long line, oriented horizontally or vertically, on which patients indicate the intensity of pruritus by marking the line at the point that corresponds to the severity of their pruritus, where the beginning of the scale refers to no pruritus ( 0 points) and the end of the scale to the most severe pruritus (10 points) $(7,8)$.

The VRS consists of a list of adjectives describing different, usually four, levels of symptom intensity, e.g. $0=$ none, $1=$ mild, $2=$ moderate and $3=$ severe/intense.

Patients were asked to record their current pruritus intensity on a VAS on a horizontal $100-\mathrm{mm}$ line, and on a four-point VRS on a questionnaire

To evaluate the influence of LSC on patients' QoL the DLQI is applied. DLQI was the first dermatology-specific QoL instrument developed in 1994. DLQI comprises 10 items, giving a sum score ranging between 0 and 30.Ten questions (Q1 to Q10) concerning symptoms, embarrassment, shopping/daily activities, clothes, and social/leisure. This validated questionnaire has been used in over 40 different skin conditions in over 80 countries and is available in over 90 languages. Its use has been described in over 1000 publications including many multinational studies. The DLQI is the most frequently used instrument in studies of randomized controlled trials in dermatology. High DLQI scores imply low QoL (9).

Individual DLQI-instrument was provided to the patients during the routine consultation. Every patient was given oral instructions on how to fill the questionnaire. The patients were asked to fill in the questionnaires at home. Completed questionnaires were returned at the next visit. The patients did not report difficulties in filling the questionnaire, which is a proof of its practical application in the routine clinical use.

Patients gave written informed consent for clinical data collection and analysis. 
The statistical analysis was performed with SPSS v.21.0 for Windows. Hypotheses were tested using $\chi^{2}$-criteria (for the descriptive profile data).Logistic regression analysis has been used to examine the independent effects of the explanatory variables on DLQI. Construct validity was tested by factor analysis. Reliability of the instrument was assessed by average inter-item correlation and Cronbach's alpha. Results with $\mathrm{p}<0.001$ were interpreted as statistically significant.

\section{RESULTS}

190 non-hospitalized adult patients 106 female (55.78\%) and 84 male (44.21\%); mean age $57.17 \pm 14.45$ years; range $22-98$ years) participated in this study. The results from measuring the degree of pruritus reported that none of patients $(0 \%)$ currently have no pruritus on the VRS ("0"), which correlated with a mean VAS value of 0 points. A total of 76 patients (40.0\%) reported having low intensity (" 1 ") pruritus (mean VAS 1.90), 70 patients (36.84\%) moderate ("2") pruritus (mean VAS 5.12), and 44 patients (23.15\%) severe (“3”) pruritus (mean VAS 8.57). The same scales were used to evaluate sleep disturbance in patients. The results were: 9 patients $(4.73 \%)$ reported currently having no sleep disturbance on the VRS ("0"), which correlated with a mean VAS value of 0.18 points. 82 patients (43.15\%) reported having low intensity ("1") pruritus (mean VAS 2.2), 90 patients (47.36\%) had moderate ("2") sleep disturbance (mean VAS 5.46), and 9 patients (4.73\%) severe (“3”) sleep disturbance (mean VAS 8.93) (fig. 1, 2).

Comparison of pruritus ratings according to gender and age (patients $<60$ years vs. $\geq 60$ years) showed no significant difference between men and women (VAS, $\mathrm{p}=0.340$; VRS, $\mathrm{p}=0.496$; nor between older ( $\geq 60$ years) and younger ( $<60$ years) patients (VAS, $\mathrm{p}=0.934$; VRS, $\mathrm{p}=0.201$.

The DLQI score for LSC was 8.58 (median 8.00; IQR 3.00-19.00). Therefore, LSH affects the QoL of

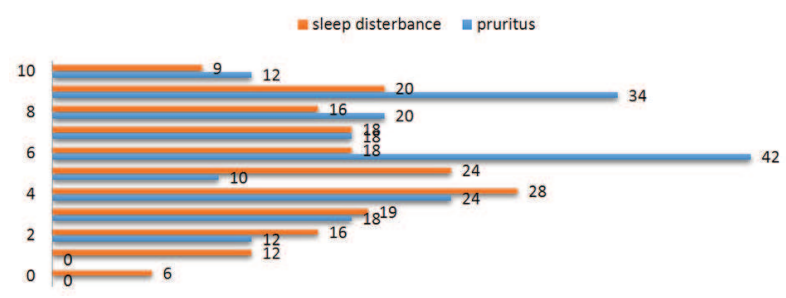

Fig. 1. Distribution according to VAS

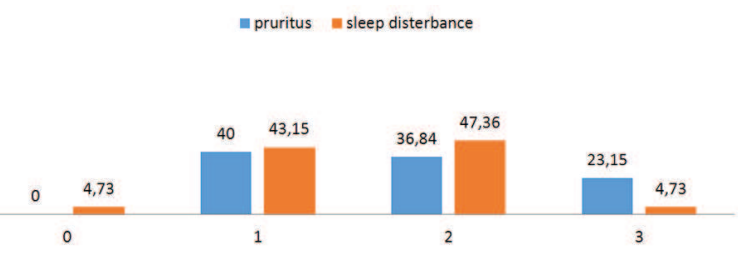

Fig. 2. Distribution according to VRS

the patients moderately. Patients who scored between 2 and 5 were only $16.8 \%$, while $57.9 \%$ of the samples had a scored between 6 and 10. Furthermore, 25.3\% of the patients scored between 11 and 20 (Table 1). Scores for the DLQI are given in Tables 2 and 3. The LSC patients scores are significantly low for all items ( $\mathrm{p}<0.001)$ except for Q1 (symptoms), Q2 (feelings), Q9 (sexual difficulties) andQ10 (treatment). Among patients with LSC, the lowest score was for Q3 (shopping), Q6 (sport) and Q8 (relationships). Scores for the six domains of DLQI were compared also (Fig. 3); DLQI scores were significantly low for all domains except for domain 1 (symptoms and feelings) anddomain 6 (treatment).

Table 1. Distribution according to DLQI- score

\begin{tabular}{lc|c} 
Range of score & Frequency & $\%$ \\
$0-1$ & 0 & 0 \\
$2-5$ & 32 & 16.8 \\
$6-10$ & 110 & 57.9 \\
$11-20$ & 48 & 25.3 \\
$21-30$ & 0 & 0.0 \\
total & $\mathbf{1 9 0}$ & $\mathbf{1 0 0}$ \\
\hline
\end{tabular}

Table 2. Scores of the answers of the 10 Questions

\begin{tabular}{|c|c|c|c|}
\hline & \multicolumn{3}{|c|}{ DLQI } \\
\cline { 2 - 4 } & median & range & p-value $^{*}$ \\
Q1 & 2.00 & $2.00-3.00$ & $<0.001$ \\
Q2 & 2.00 & $1.00-2.00$ & $<0.001$ \\
Q4 & 0.00 & $0.00-1.00$ & $<0.001$ \\
Q5 & 1.00 & $0.00-1.00$ & $<0.001$ \\
Q6 & 1.00 & $0.00-1.00$ & $<0.001$ \\
Q7 & 0.00 & $0.00-0.00$ & 0.182 \\
Q8 & 1.00 & $0.00-1.00$ & $<0.001$ \\
Q9 & 0.00 & $0.00-0.00$ & 0.155 \\
Q10 & 1.00 & $0.00-2.00$ & $<0.001$ \\
\hline \hline
\end{tabular}

Scripta Scientifica Medica, vol. 48, No. 2, 2016, pp. 50-54 Medical University of Varna 
Table 3. Six dimensions' scores

\begin{tabular}{lc|c|c|} 
& \multicolumn{3}{c|}{ DLQI } \\
\cline { 2 - 4 } & Median & range & p-value* \\
$\begin{array}{l}\text { 1. Symptoms \& } \\
\text { feelings }\end{array}$ & 4.00 & $3.00-5.00$ & $<0.001$ \\
2. Daily activities & 1.00 & $0.00-2.00$ & $<0.001$ \\
3. Leisure & 0.00 & $0.00-1.00$ & $<0.001$ \\
4. Work/school & 1.00 & $0.00-1.00$ & $<0.001$ \\
$\begin{array}{l}\text { 5. Personal } \\
\text { relationship }\end{array}$ & 1.00 & $0.00-2.00$ & $<0.001$ \\
6. Treatment & 2.00 & $1.00-2.00$ & $<0.001$ \\
\hline \hline
\end{tabular}

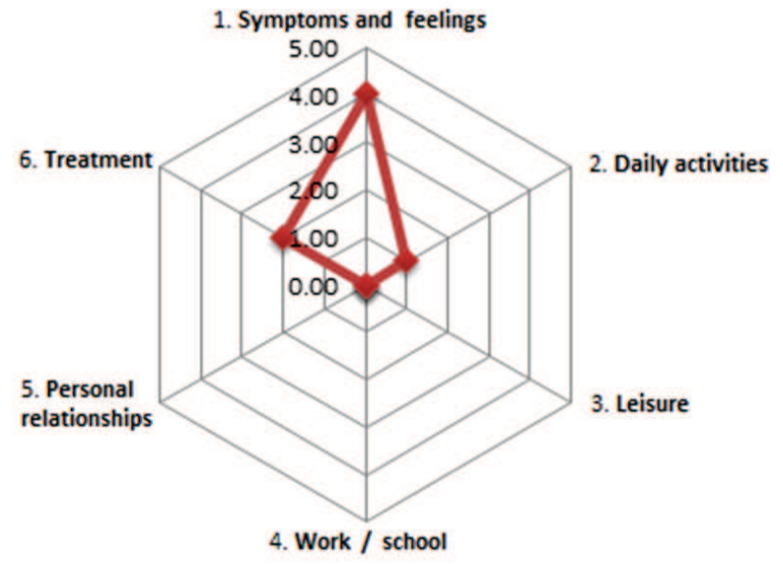

Fig. 3. Distribution of patients' score according DLQI-domains

\section{DISCUSSION}

Pruritus is a major symptom of a huge variety of dermatoses. It causes a scratch response, starting the itch-scratch cycle, which in turn aggravates the inflammatory response and exacerbate disease severity (4). Many authors have underlined that this often ignored symptom is chronic in nature with a huge impact on the QoL of the patient (10). Patients reported that they try to control the act of scratching during the day but not during sleep. The most common cases of insomnia are impossibility to sleep, awakening because of a feeling of itch and difficult to fall asleep again (11). This gave us a reason to examine not only the intensity of the itch but its effect on sleep quality and respectively on QoL. As Sonja Grundmann (2011) mentioned there are no standardized methods for documenting itching (12). A study of Ständer $S$ and associates (2006) emphasized that the feeling of itching is strictly subjective and depends on the stress, anxiety and current conditioning (13). To assess the severity of itching and sleep disturbance we used the scales - VAS and VRS (12). Using the same scales Sanjana and Fernandez (1995) reported that $69.5 \%$ of the patients included in their study have VAS $=6-10$, and the other $30,5 \%$ have VAS $=5$ (14). At the same time Rajalakshimi and associates (2011) in a study of 105 patients with ano-genital localized neurodermatitis reported the following distribution - 21.9\% VAS $=8,20 \%$ VAS $=6$ and $16.2 \%$ VAS $=5$ (15). The lower values of VAS, obtained in our study can be explained with the fact that we included patients with different localization of the pathological process not just ano-genital ones.

In the literature we found evidence on an objective measurement of the degree of itching and sleep disturbance in patients with LSH in the ano-genital region $(14,15)$ but not in other localizations which entitles us to believe that this is the first objective documenting and measuring study on itching and sleep disturbance in patients with LSH.

Another aspect of our study was comparing the severity of pruritus and sleep disturbance with the results obtained from answers to - DLQI. (Specially answer to question1- symptoms and domain 1 (symptoms and feelings)

In previous studies by other authors with a 30 item questionnaire DLQI detected a significant impact on the subjective symptoms (Q1) and sexuality (Q9) (16,17). Jin-Gang-An et al (2013) conducted a study involving 149 patients diagnosed with LSH. The authors reported deterioration of QoL in 32.9\% of the included patients with DLQI -9.34 (18).Our results show that, QoL is negatively influenced in all enrolled patients, 190 (100\%) with moderately affected QoL in - 57.9\%. The mean DLQI score in our study was 8.58. This correlates with the export of Jin-GangAn et al (2013) (18) and Ermertcan et al (2011) (19) data. Severe itching is a prominent feature of LSC.Results from our study showed that Question 1 (symptoms) and domain 1(symptoms and feelings) had the biggest impact on LSC patients (mean 2.00, range $1.00-2.00)(\mathrm{p}<0.001)$ which is supported by the results from Iman Hamed El-Maadawy et al (2014) (20).

This is the first study in Bulgaria, which aims to measure the impact of pruritus on QoL of patients with LSC for both male and female patients. 


\section{CONCLUSION}

LSC is extremely pruritic dermatological condition. Itch is a major symptom associated with LSC and affects negatively the QoL of the patients. Therefore, a study of their self-reported health status alongside with the clinical investigation is a necessary precondition for successful therapeutic results. There was a limitation in this study as far as pruritus and QoL of patients were assessed once and not in dynamics, before and after treatment. This could be an issue in the planning of a future research study.

\section{REFERENCES}

1. TorelloLotti GB. Francesca Prignano. Prurigonodularis and lichen simplex chronicus. Dermatologic Therapy 2008; 21:42-46

2. Martin-Braufau R, Corbalan-Berna J, RamirezAndrero A. Braufau-Redondo C. LimlnanaGras R. Personality differences between patients with lichen simplex chronicus and normal population:A study of pruritus. Eur.J.Dermatol 2010; 20(3):359-363

3. Yosipovitch G, Samuel LS. Neuropathic and psychogenic itch.DermatolTher.2008; 21(1):32-41.

4. Kini SP, DeLong LK, Veledar E, McKenzie-Brown AM, Schaufele M, Chen SC. The impact of pruritus on quality of life: the skin equivalent of pain. Arch Dermatol. 2011; 147(10):1153-1156

5. Lilit Garibyan, Curtis G. Rheingold, and Ethan A. Lerner Understanding the pathophysiology of itch Dermatol Ther2005; 23(4):591-599

6. WHO. WHOQOL-BREF - Instructions, Administration, Scoring and Generic Version of the AssessmentWHO. Geneva; 1996-5.

7. Phan NQ, Blome C, Fritz F, Gerss J, Reich A, Ebata T, et al. Assessment of pruritus intensity: prospective study on validity and reliability of the visual analogue scale, numerical rating scale and verbal rating scale in 471 patients with chronic pruritus. Acta Derm Venereol 2012; 92: 502-507.

8. Reich A, Heisig M, Phan NQ, Taneda K, Takamori K, Takeuchi S, et al. Visual analogue scale: evaluation of the instrument for the assessment of pruritus. Acta Derm Venereol 2012; 92: 497-501.

9. http://sites.cardiff.ac.uk/dermatology/quality-of-life/ dermatology-quality-of-life-index-dlqi
10. Weisshaar EW, Dalgard F. Epidemiology of itch: adding to the burden of skin morbidity. acta Derm venereol 2009; 89: 339-350

11. Rafet Koca, Remzi Altin, Numan Konuk, H. Cevdet Altinyazar, and Levent Kart Sleep disturbance in patients with lichen simplex chronicus and its relationship to nocturnal scratching: a case control study Southern Medical Journal May 2006: 99(5):482-5

12. Grundmann Sonja, Ständer Sonja, Chronic Pruritus: Clinics and Treatment Ann Dermatol. 2011 Feb;23(1):1-11

13. Ständer S, Streit M, Darsow U, Niemeier V, Vogelgsang M, Ständer H, et al. Diagnostic and therapeutic procedures in chronic pruritus. J Dtsch Dermatol Ges 2006;4:350-370

14. Sanjana VD, Fernandez RJ. Lichen simplex chronicus: A psychocutaneous disorder? Indian J Dermatol Venereol Leprol 1995;61:336-8

15. R Rajalakshmi, Devinder Mohan Thappa, Telanseri J Jaisankar, Amiya Kumar Nath Lichen simplex chronicus of anogenital region: A clinico-etiological study IJDVL 2011;77(1):28-36

16. Fortune DG, Main CJ, OSullivan TM., Griffithis CEM., Quality of life in patients with lichen simplex chronicus: the contribution of clinical variables and pruritus- specific stress British Journal of Dermatology 2002, 137, 755-760

17. Root, S Kent, G \&Al Abadie, MSK The relationship between disease severity, disability and psychological distress in patients with lichen simplex chronicus Dermatology 2013; 189; 234-237

18. Jin Gang An, Yan-Ting Liu, Sheng-Xiang Xiao, Jun-Min Wang, Song-Mei Geng, Ying-Ying Dong. Quality of Life of Patients with Neurodermatitis. International Journal of Medical Sciences 2013, 10 593-598

19. Ermertcan AT, Gengolgan G, Temelsan G, Horasan GD, Deveci A, Oztuk F. Sexual dysfunction in female patients with neurodermatitis J Androl 2011; 32(2); 165-169

20. Iman Hamed El-Maadawy, Amal Ahmad El-Ashmawy Impact of some chronic dermatoses on Quality of Life of Egyptian patients in Delta Region Egyption Dermatology Online Journal 2014 June 10(1);2 